\title{
AN EXAMPLE INVOLVING BAIRE SPACES
}

\author{
H. E. WHITE JR.
}

ABSTRACT. If $2^{\boldsymbol{X}_{0}}=\boldsymbol{N}_{1}$, then there is a regular, Hausdorff space $Y$ such that (1) every subspace of $Y$ is a Lindelöf, Baire space, (2) $Y$ is a homogeneous space, and (3) $Y \times Y$ is of the first category.

In this note we show, using a minor modification of an argument of Oxtoby [5, pp. 163-164], that, if $2^{\boldsymbol{N}_{0}}=\boldsymbol{N}_{1}$, then there is a dense subspace $Y$ of $(R, \mathcal{T})$, where $\mathcal{T}$ denotes the density topology on the real line $R$, which has the properties listed in the abstract. The space $(R, \mathcal{T})$ is a completely regular, Hausdorff, Baire space with the property that

(1.1) a subset $A$ of $R$ is $\mathcal{J}$-nowhere dense if and only if the Lebesgue measure of $A$ is zero.

The density topology $\mathcal{T}$, which consists of all Lebesgue measurable subsets of $R$ having density 1 at each of their points, was introduced in [3] and studied in [2]; see $\$ 2$ of [6] for more of its properties.

We shall denote the Euclidean topology on $R$ by $\mathcal{E}$. For any topological space $(X, \mathcal{U})$, we denote by $\mathcal{U} \times \mathcal{U}$ the product topology on $X \times X$ induced by $\mathcal{U}$. And, for any family $\mathfrak{F}$ of subsets of $R$ and any subset $A$ of $R$, we denote $\{F \cap A: F \in \mathfrak{F}\}$ by $\mathcal{F} \cap A$.

Proposition. Suppose $G$ is an additive subgroup of $R$ of positive Lebesgue outer measure such that $G$ is of the first category in $(R, E)$. Then $(G, \mathcal{J} \cap G)$ is a completely regular, Hausdorff space such that (1) every subspace of $G$ is a Baire space, (2) $G$ is bomogeneous, and (3) $G \times G$ is of the first category (in $G \times G)$.

Proof. It is easily seen that $G$ is dense in $(R, \mathcal{J})$. Next, there is a dense $G_{\delta}$ subset $H$ of $(R, \mathcal{E})$ such that $G \cap H=\varnothing$. Let $K=$ $\{(x, y) \in R \times R: x-y \in H\}$. Then $K$ is a $G_{\delta}$ subset of $(R \times R, \xi \times \mathscr{G})$. And (see [4]), if $A, B$ are Lebesgue measurable subsets of $R$ of positive measure, then $K \cap(A \times B) \neq \varnothing$. Hence $K$ is a dense $G_{\delta}$ subset of $(R \times R$,

Presented to the Society, July 13, 1973; received by the editors August 16, 1973. AMS (MOS) subject classifications (1970). Primary 54D99, 54G20.

Key words and phrases. Baire space, density topology, homogeneous space. 
$\mathcal{T} \times \mathfrak{T})$. Because $G \times G$ is dense in $(R \times R, \mathcal{T} \times \mathfrak{T})$ and $(G \times G) \cap K=\varnothing$. $G \times G$ is of the first category.

Because every isometry of $(R, \mathcal{E})$ is a homeomorphism of $(R, \mathcal{T})$ onto itself, and $G$ is an additive subgroup of $R,(G, \mathcal{T} \cap G)$ is homogeneous.

If $A \subset R$ and $A$ is of the first category in $(R, \mathcal{T})$, it follows from (1.1) and the definition of $\mathfrak{T}$ that $(A, \mathfrak{T} \cap A)$ is discrete. It follows easily from this, that every subspace of $(R, \mathcal{T})$ is a Baire space; hence (1) holds.

In the following, we denote Lebesgue measure on $R$ by $m$ and the set of all rational numbers by $Q$. For any subset $A$ of $R$ and any $x, y$ in $R$, we denote by $x A+y$ the set $\{x a+y: a \in A\}$.

Construction of the space $Y$ that is described in the abstract. Suppose $2^{\boldsymbol{X}_{0}}=\boldsymbol{K}_{1}$. Let $Z$ denote a dense $G_{\delta}$ subset of $(R, \mathcal{E})$ such that $0 \notin Z$ and $m(Z)=0$. Suppose $\left(F_{a}\right)_{a<\omega_{1}}$ is the family of all $\tilde{E}$-Borel sets of measure zero, and suppose $F_{0}=Z$.

Let $Y_{0}=\{0\}$. Suppose that, for some $\alpha, 0<\alpha<\omega_{1}$, we have defined $\left(Y_{\beta}\right)_{\beta<a}$ so that, for each $\beta, 0 \leq \beta<\alpha, Y_{\beta}$ is a countable subset of $R$. Let $Y^{\alpha}=\bigcup\left\{Y_{\beta}: \beta<\alpha\right\}$ and $Z^{\alpha}=\bigcup\left\{q F_{\beta}+y: q \in Q, \beta<\alpha, y \in Y^{\alpha}\right\}$. Because $m\left(Z^{\alpha}\right)=0$, we may choose $b_{a}$ in $R \sim Z^{\alpha}$. Let $Y_{a}=\left\{q b_{a}+y: q \in Q\right.$, $\left.y \in Y^{a}\right\}$. Finally, let $Y=\bigcup\left\{Y_{a}: a<\omega_{1}\right\}$. It is easily seen that $Y$ is a rational vector subspace of $R$. We shall now show that

$$
\left(Y_{\alpha} \sim Y^{\alpha}\right) \cap\left[\bigcup\left\{F_{\beta}: \beta<\alpha\right\}\right]=\varnothing \quad \text { for } 0<a<\omega_{1} .
$$

Suppose $x \in\left(Y_{\alpha} \sim Y^{\alpha}\right) \cap\left[\bigcup\left\{F_{\beta}: \beta<\alpha\right\}\right]$, where $0<\alpha<\omega_{1}$. Then there are $y<\alpha, q$ in $Q$, and $y$ in $Y^{\alpha}$ such that $x \in F_{\gamma}$ and $x=q b_{\alpha}+y$. Because $x \notin Y^{a}, q \neq 0$. Then $b_{a} \in q^{-1}\left[F_{\gamma}+(-y)\right] \subset Z^{a}$, contradicting the choice of $b_{a}$.

It follows from (1.2) that (a) $|Y \cap A| \leq \boldsymbol{x}_{0}$ for every subset $A$ of $R$ of Lebesgue measure 0 , and (b) $Y \cap Z=\varnothing$. From (a) it follows that, because $|Y|>\boldsymbol{\kappa}_{0}, Y$ has positive Lebesgue outer measure. And it follows from (a), (1.1), and the fact that $(R, \mathcal{T})$ obviously satisfies the countable chain condition, that $(Y, \mathcal{T} \cap Y)$ is hereditarily Lindelöf.

Remarks. (1) $Y$ is a totally nonmeagre space $[1$, p. 252] such that $Y \times Y$ is not a Baire space.

(2) It follows from $[6,2.1 .2]$ that Blumberg's theorem does not hold for $Y$.

(3) To show that $Y$ is completely regular we do not need to know that $(R, \mathfrak{T})$ is completely regular; it is sufficient to know that $(R, \mathfrak{T})$ is a regular, Hausdorff space. This latter statement is rather easy to prove. 
(4) The space $Y$ is not homeomorphic with the space $Z$ constructed on p. 164 of [5] because $Z$ is extremally disconnected and $Y$ is not.

(5) If we replace the continuum hypothesis with the following hypothesis, then the argument used in constructing $Y$ can be used, with only minor modifications, to construct a set $G$ satisfying the hypothesis of the proposition.

If $\mathcal{F}$ is a family of subsets of $R$ of Lebesgue measure 0 such that $|\mathfrak{F}|<2^{\aleph_{0}}$, then the interior Lebesgue measure of $\bigcup \mathcal{F}$ is 0 .

(6) Dr. R. M. Solovay has stated (in a letter) that (5) implies that if Martin's axiom holds, the $R$ contains a subgroup satisfying the hypothesis of the proposition. He has stated further that it is consistent with $\mathrm{ZF}+\mathrm{AC}$ that there is no subgroup of $R$ which satisfies the hypothesis of the proposition.

(7) If $G$ satisfies the hypothesis of the proposition, then $(G,+, \mathcal{T} \cap G)$ is not a topological group. For, if $U$ is an element of $\mathcal{J}$ containing 0 such that $0 \notin \mathcal{E}$-int $(\mathcal{E}$-cl $U)$, then there is no $V$ in $\mathcal{T}$ such that $0 \in V$ and $\{x-y: x, y \in V \cap G\} \subset U \cap G$.

\section{REFERENCES}

1. N. Bourbaki, Elements of mathematics: General topology. Part 2, AddisonWesley, Reading, Mass., 1966.

2. C. Goffman, C. J. Neugebauer and T. Nishiura, Density topology and approximate continuity, Duke Math. J. 28 (1961), 497-505. MR 25 \#1254.

3. C. Goffman and D. Waterman, Approximately continuous transformations, Proc. Amer. Math. Soc. 12 (1961), 116-121. MR 22 \#11082.

4. H. Helson, Remark on measures in almost-independent fields, Studia Math. 10 (1948), 182-183. MR 10, 600.

5. J. C. Oxtoby, Cartesian products of Baire spaces, Fund. Math. 49 (1960/61), 157-166. MR 25\#4055; $26 \# 1543$.

6. H. E. White, Jro, Topological spaces in which Blumberg's theorem holds, Proc. Amer. Math. Soc. 44 (1974), 454-462.

Current address: 351 Eldon Avenue, Columbus, Ohio 43204 